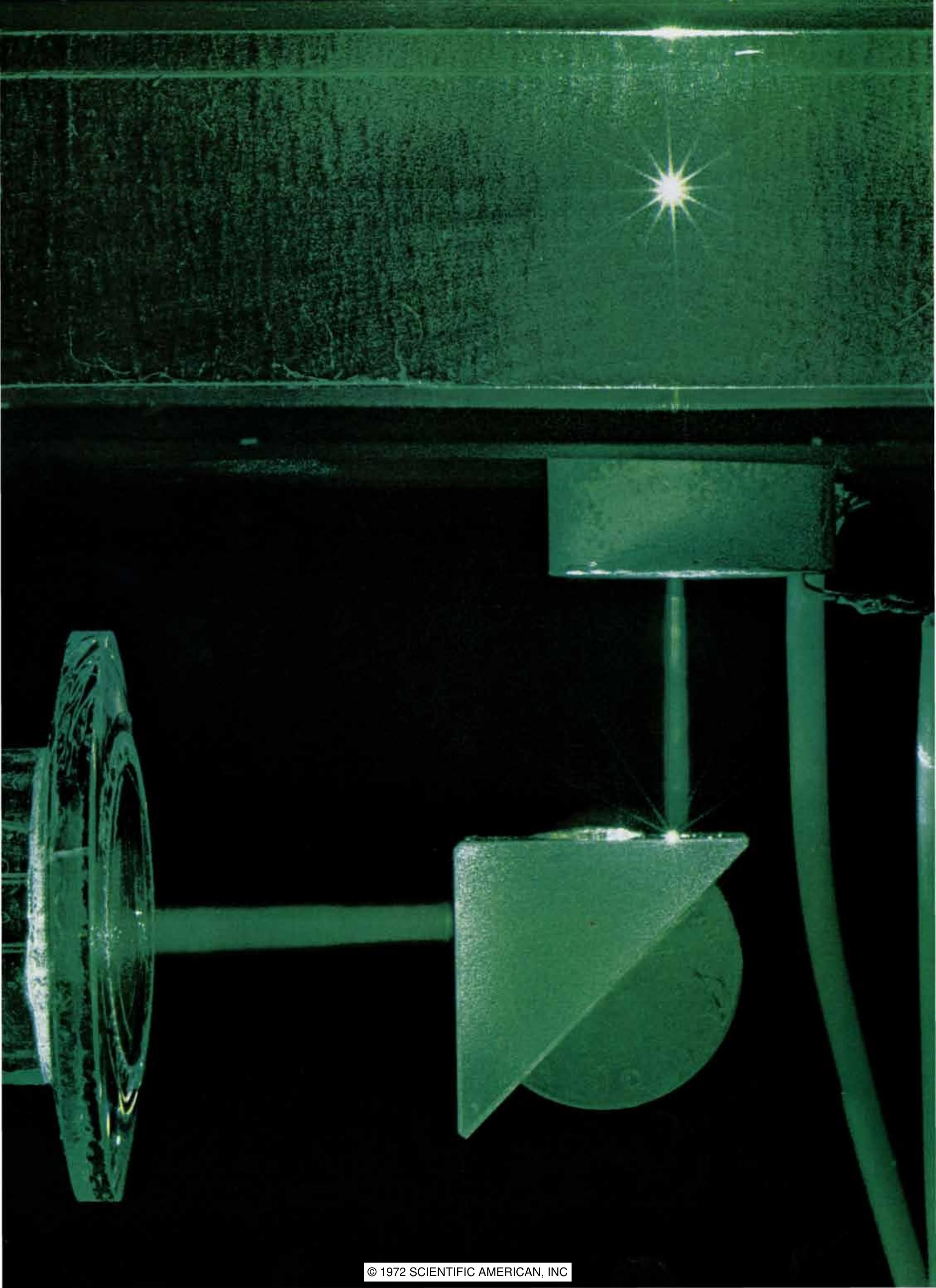




\title{
THE PRESSURE OF LASER LIGHT
}

\author{
The forces exerted by a focused beam of laser light are strong \\ enough to push tiny particles around freely in various mediums. \\ Several applications based on this recent finding are proposed
}

\author{
by Arthur Ashkin
}

$\mathrm{I}$ is common knowledge that light carthat light also carries momentum. When we sit in sunlight, we are quite conscious of the heat from the light but not of any push. Nonetheless, it is true that whenever ordinary light strikes an object, the collision gives rise to a small force on the object. This force is called radiation pressure.

The possibility that light could exert pressure goes back to Johannes Kepler, who in 1619 postulated that the pressure of light is what causes comets' tails to always point away from the sun. The corpuscular theory of light introduced by Isaac Newton made the idea of radiation pressure more plausible and stimulated many experimental attempts to measure it. During the 18th and 19th centuries all such attempts to detect the postulated pressure failed to reveal any force that could not be attributed to convection in the air caused by heat. In 1873 Sir William Crookes thought he had discovered radiation pressure in a partly evacuated chamber, only to find that he had invented the radiometer. (The little rotating-vane toy seen in many opticians' windows is a radiometer. It responds to the forces of molecular bombardment on surfaces heated by light rather than to radiation pressure. These thermal forces ries energy. Less obvious is the fact

are called radiometric forces.) It was also in 1873 that James Clerk Maxwell predicted the magnitude of radiation pressure based on his new theory of electromagnetic waves. The predicted pressure was extremely small for ordinary light sources. In Crookes's experiment, for example, it was four or five orders of magnitude smaller than the observed radiometric forces.

The existence of radiation pressure, free of disturbing thermal effects, was finally demonstrated experimentally around the turn of the century by Ernest F. Nichols and G. F. Hull in the U.S. and by P. N. Lebedev in Russia. In both experiments the radiation pressure exerted by a light source was detected by a twisting motion of a vane suspended by a fine fiber in a high vacuum. The magnitude of the force measured in this way confirmed Maxwell's prediction. Commenting on the subject of radiation pressure in his presidential address to the British Physical Society in 1905, John H. Poynting said: "A very short experience in attempting to measure these light forces is sufficient to make one realize their extreme minuteness-a minuteness which appears to put them beyond consideration in terrestrial affairs." Poynting's view of the situation held true until just recently. The experiments described

OPTICAL LEVITATION of a very small particle by means of a vertically directed beam of green laser light is demonstrated in the photograph on the opposite page. The particle, a transparent glass sphere 20 microns in diameter, is shown levitated in air about a centimeter above a glass plate by the 250 -milliwat beam. Although the particle is barely visible to the unaided eye, it scatters enough laser light to sparkle brilliantly in this experiment; the bright spikes radiating from its image in the photograph are caused by diffraction around the blades of the diaphragm inside the camera. Smoke was blown in the path of the laser beam to make it visible after it left the focusing lens (lower left) and was reflected upward by a prism (lower right) before entering the glass enclosure. The structure with the two wires attached is a piezoelectric ceramic cylinder used to vibrate the particle momentarily in order to break the weak van der Waals forces holding it to the surface of the glass plate. The interference pattern visible against the back wall of the enclosure is caused by light scattered at an angle of about 90 degrees by the particle. here, which show that the phenomenon of radiation pressure does indeed merit "consideration in terrestrial affairs," could not have been conducted before the invention of the laser in 1960.

The laser has opened up new fields of optical research and rejuvenated old ones. The special features of laser light that have brought about these changes are its high degree of spectral purity and its spatial coherence. These properties make it possible, among other things, to focus a laser beam to a spot with a radius close to the theoretical limit of one wavelength. Thus even with a power of a few watts one can obtain a light intensity at one wavelength that is some 10,000 times greater than the intensity available from the entire visible spectrum at the surface of the sun! Moreover, a laser beam operates with a simple, mathematically perfect, well-controlled intensity profile called a transverse mode. The most useful mode has a cross section with a simple Gaussian, or bell-shaped, energy distribution. Another important achievement has been the development of tunable lasers, with which one can select the wavelength of the light at will.

Radiation pressure has recently been reexamined in the light of these new laser sources and has been found to be a strong effect. Indeed, the forces exerted by laser sources have been shown to be large enough to move small particles around freely in various mediums. Accelerations as large as a million $g$ (a million times the acceleration of gravity) are attainable on a continuous basis for tiny macroscopic particles as well as for individual atoms and molecules. These findings have given rise to new applications based on the physical motion of small particles driven by radiation pressure. This rather exotic force has also been found to have a number of unique 


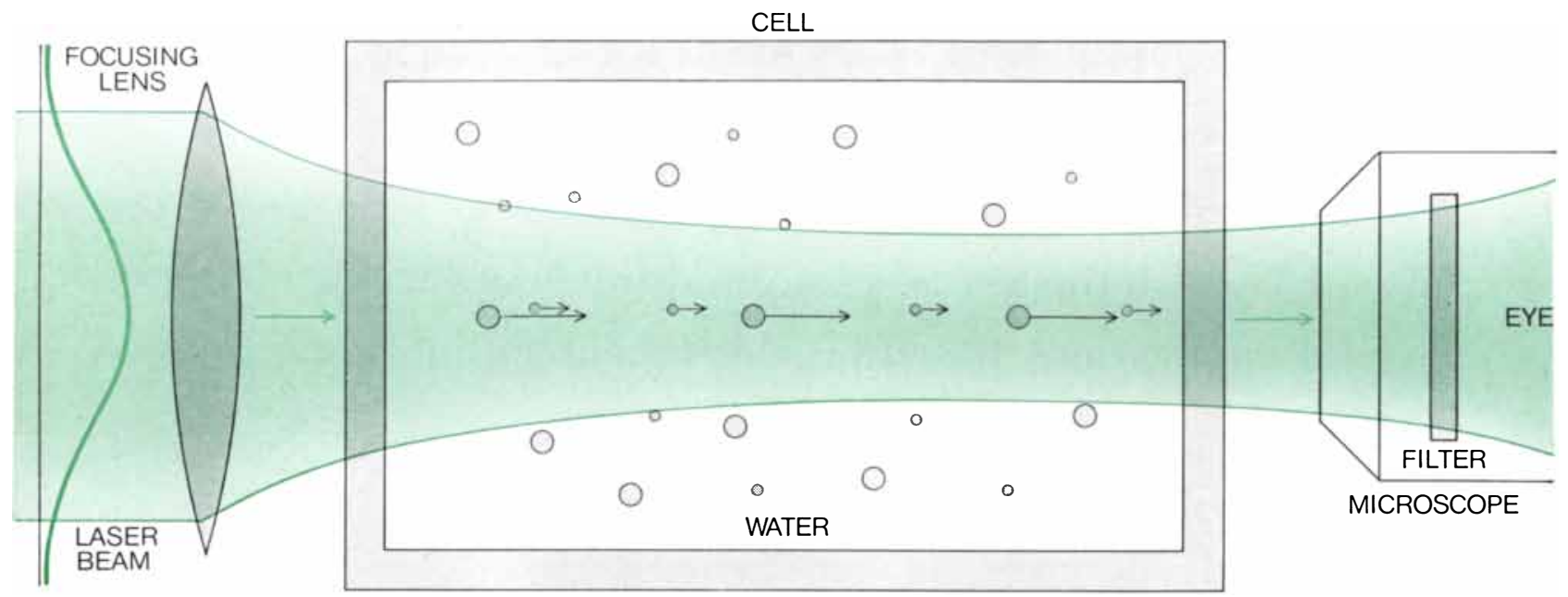

HORIZONTALLY FOCUSED LASER BEAM is used to push transparent plastic spheres of two sizes, 2.5 microns and .5 micron in diameter, through a water-filled cell. The larger spheres are observed to move faster than the smaller ones (left). The motions of the particles are viewed from beyond the far end of the cell with a microscope and a filter that absorbs the laser light. It is possible in this way to observe the trapping of a particle at the far face of the cell (right). A sphere originally trapped at the center of the beam will wander off randomly when the beam is turned off and will return directly to the beam center when the beam is turned on features, some not realized before and some resulting from the nature of laser light itself. A few of the possible applications are the separation of particles in liquids, the optical levitation of particles in air and vacuum, the high-velocity acceleration of electrically neutral particles, the separation of isotopes and the analysis of atomic beams.

The starting point of all this work was simply a hunch on the part of the author that the radiation pressure of laser light could be a large effect even with the modest power available from continuously operating lasers. The following simple order-of-magnitude calculation was made. Suppose a watt of continuous green laser light (with a wavelength of about .5 micron) is focused to a spot with a radius equal to the wavelength of the light. What is the force exerted on a small spherical particle of the same size placed at the focus? Assuming that the particle acts as a perfect mirror, one computes a force of approximately $10^{-3}$ dyne. If the particle has unit density, its mass is about $10^{-12}$ gram. By Newton's law this result implies an acceleration of a million times the acceleration of gravity-obviously a large acceleration!

Such calculations strongly suggested trying an experiment to move small particles with light pressure. Highly reflecting metal particles were considered, but even with a reflectivity of 98 percent the residual absorption of 2 percent would surely cause severe heating, possibly even melting. Here again one is up against the thermal problems that were the bane of early experimenters. One of the prices of increased light intensity is increased heating. The solution seemed clear: to use transparent dielectric (nonconducting) particles. Although the reflectivity of bare dielectric surfaces such as glass or plastic is relatively low, the forces are still considerable.

In the following experiment, carried out 1 in mylaboratory at the Bell Telephone Laboratories, small, precisely spherical, transparent plastic particles made by the Dow Chemical Company were used. These spheres are customarily supplied in water and therefore it seemed natural to try the first experiments in water. Besides, the high optical transparency and high thermal conductivity of water would further help to keep the particles cool. A few drops of water containing some particles were accordingly placed in a glass cell under a microscope and a green argon-ion laser beam was focused through the cell from below. By wearing special eyeglasses that absorb only the laser light it was possible to protect one's eyes and still observe the particles by ordinary light. The laser beam was visible anyway by virtue of a mild yellow fluorescence that it induces in almost anything it hits. Hence it turned out to be a simple matter to see the beam, focus it and manipulate it by moving the focusing lens. Since the plastic spheres had the same density as the water, they remained essentially stationary as fixed targets. When a vertically directed Gaussian-mode laser beam with a power of about 10 milliwatts and a diameter of 15 microns was directed at a particle a few microns across, the particle began to rise at a constant velocity of some $10 \mathrm{mi}-$ crons per second until it reached the top surface of the cell. (One expects a particle moving in a viscous medium under the influence of a constant force to travel at a constant velocity, in accordance with Stokes's law.)

So the particles moved. Several questions remained. For example, is the motion really due to radiation pressure or is it due to some residual thermal effects such as convection or photophoresis? In the case of convection the entire liquid would move, carrying the particles along. In the case of photophoresis single particles would be heated asymmetrically by the light and would as a result move through the surrounding medium.

To answer these questions a second experiment was performed with two modifications. Particles of two sizes, 2.5 microns and .5 micron in diameter, were used simultaneously and the beam was passed through the cell horizontally. Thus if the liquid through which the beam passes were heated, the liquid would tend to rise rather than flow along the axis of the light beam. Again the particles moved in the direction of the light beam, only now the larger particles moved rapidly past the slower-moving small particles [see illustration above]. This observation in itself ruled out convection as a cause of the motion.

In addition it was observed that when a particle hit the far face of the cell, it remained there, seemingly trapped in the center of the beam. If the beam was interrupted, the particle began to wander off center. If the light was turned on again when the particle was in the fringes of the light, it was immediately drawn 


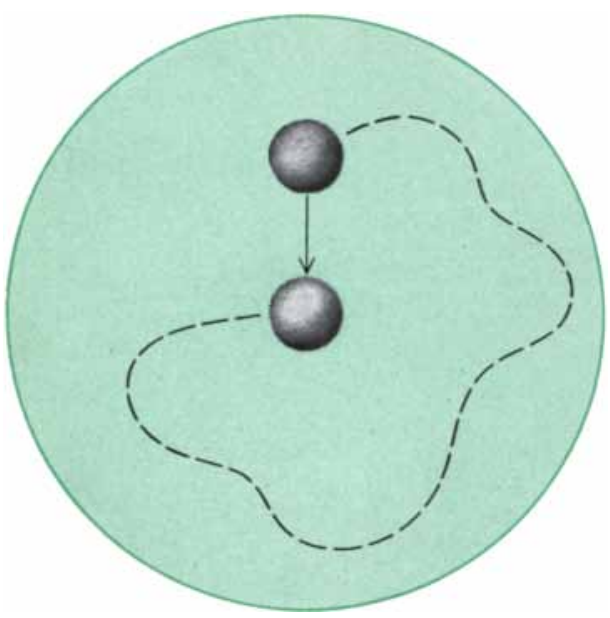

again. The laser beam in these experiments operates with an intensity profile (called a transverse mode) that has a cross section with a simple Gaussian energy distribution (colored bell-shaped curve at extreme left).

back to the center of the beam. Similarly, if a particle in the bulk of the liquid was illuminated off center near the fringes of the beam, it was observed not only to move in the direction of the beam but also to be drawn into the center of the beam where the light intensity was a maximum [see bottom illustration on this page]. There seemed no escaping the conclusion that there was not only a force along the beam but also a transverse force drawing particles into the center of the light beam.

This observation helped to rule out photophoresis as the cause of the observed motion of the particles. In photophoresis the force on the hot side of a particle is larger than it is on the cool side and an off-center particle would be pushed out of the beam, contrary to what was observed. Also in photophoresis a partially transparent particle heated by the beam would tend to move backward with respect to the direction of the light, again contrary to the observation. (This backward motion, called negative photophoresis, can be readily seen if one shines laser light on partially transparent smoke particles floating in air. Its explanation is based on the converging effect of light passing through the particle, which makes the downstream side of the particle the hot side.)

It now remains to show how radiation pressure accounts for the observations. The magnitude of the force on the plastic sphere can be calculated by adding the effects of all the light rays hitting it. Each ray, as it is partially reflected and refracted at the surfaces of the sphere, carries off some of the light in different directions and gives rise to a contribution to the net force. From the value of the force when the particle is on the beam axis and from Stokes's law one can compute the expected velocity of the sphere through the water. The computed velocity agrees with the measured velocities within experimental error. This agreement alone indicates that no other large forces are operative.

Similarly one can show how the transverse components of the force act on a sphere located off axis in a Gaussian beam. Imagine a beam of light striking a plastic sphere that has a higher index of refraction than the surrounding water [see top illustration on next page]. Consider a typical pair of light rays, $a$ and $b$, situated symmetrically with respect to the center of the sphere. Disregard surface reflections and consider only the transmitted part of each ray, since this usually is the strongest part and makes the principal contribution to the force. The rays are bent in such a way as to give rise to two forces, $F_{a}$ and $F_{b}$, located along the direction of the momentum changes for the rays. Since ray $a$ is stronger than ray $b$, force $F_{a}$ is greater than force $F_{b}$, and one would expect a net transverse force pulling the highrefractive-index sphere in toward the center of the beam, where the intensity is a maximum. That is what happens.

There is an obvious extension of this argument. If the sphere has a lower index of refraction than its surrounding medium, forces $F_{a}$ and $F_{b}$ would reverse, and such a sphere should be pushed out of the beam, away from the maximum light intensity. This also happens. After a frustrating search for low-refractive-index particles in a highrefractive-index liquid it occurred to me to use tiny air bubbles in a viscous liquid such as glycerin. After all, bubbles behave like particles, and there is a dis- continuity in refractive index at the surface of a bubble, so that light should exert a force on it. Furthermore, a bubble represents the ultimate in a nonabsorbing particle, and it has the lowest possible index of refraction. Small bubbles of the desired size can be generated by using a blender to agitate glycerin diluted with water. When the bubbles are hit with a laser beam, they move as expected, forward and out of the light beam. They avoid the light, in contrast to the behavior of high-refractive-index particles.

The picture of how the transverse forces arise is now complete. An important consequence of this picture is that it implies one can shape light beams that will trap particles by radiation pressure alone, forming what might be called an "optical bottle." A high-index particle placed at a certain point in such a nonmaterial container should be in stable equilibrium, since any displacement would result in an optical restoring force. When an actual light configuration of this type was set up in a water cell, the particles drifting into the fringe fields were sucked into the trap, where they remained stationary [see bottom illustration on next page]. The fact that a particle was really trapped was demonstrated by intercepting one beam for a moment. The particle pushed by the second beam rapidly began to escape, whereupon the first beam was turned on again. The particle then returned to the equilibrium point, only more slowly, since it was operated on by the differential restoring force. The procedure could be repeated many times with either beam being interrupted.

Liquid has clearly proved to be a useful experimental medium for studying radiation pressure. Liquid may also be the preferred medium for many applications of radiation pressure, such as schemes for particle separation. Differ-

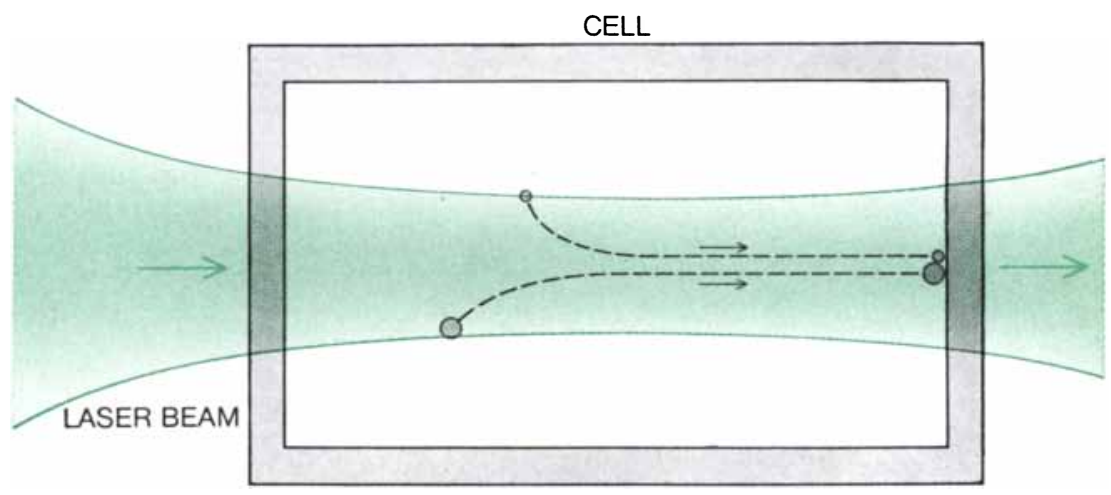

PARTICLES ILLUMINATED NEAR THE EDGE of a Gaussian-mode laser beam are not only pushed in the direction of the light but also drawn toward the center of the beam. 

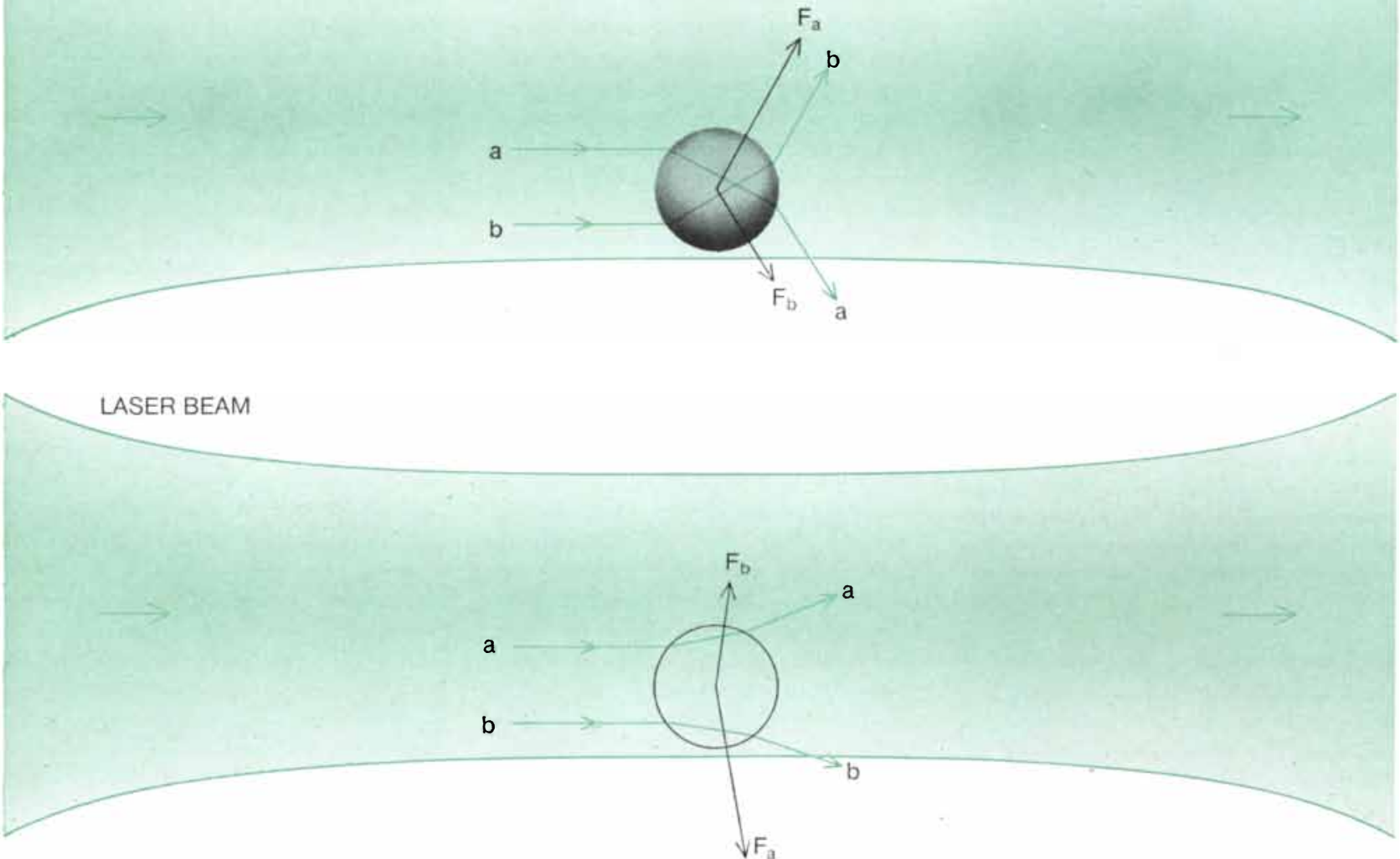

INDEX OF REFRACTION of a particle with respect to its surroundings determines whether the laser beam will push it toward or away from the center of the beam. The top diagram shows the forces associated with the refraction of a pair of typical light rays $(a, b)$ as they pass through an off-center sphere that has a higher index of refraction than its surroundings. Force $F_{a}$ is greater than force $F_{b}$; therefore there is a net transverse component of force toward the bean center. The bottom diagram shows the corresponding forces for an off-center sphere with a lower index of refraction than its surroundings. The refraction of the light rays is reversed; since $\boldsymbol{F}_{a}$ is still greater than $\boldsymbol{F}_{b}$ but is now pointed away from the center, there is a net transverse force pushing the particle away from the center. In both cases $\boldsymbol{F}_{a}$ and $\boldsymbol{F}_{b}$ have components pushing the sphere in the direction of the light as well.

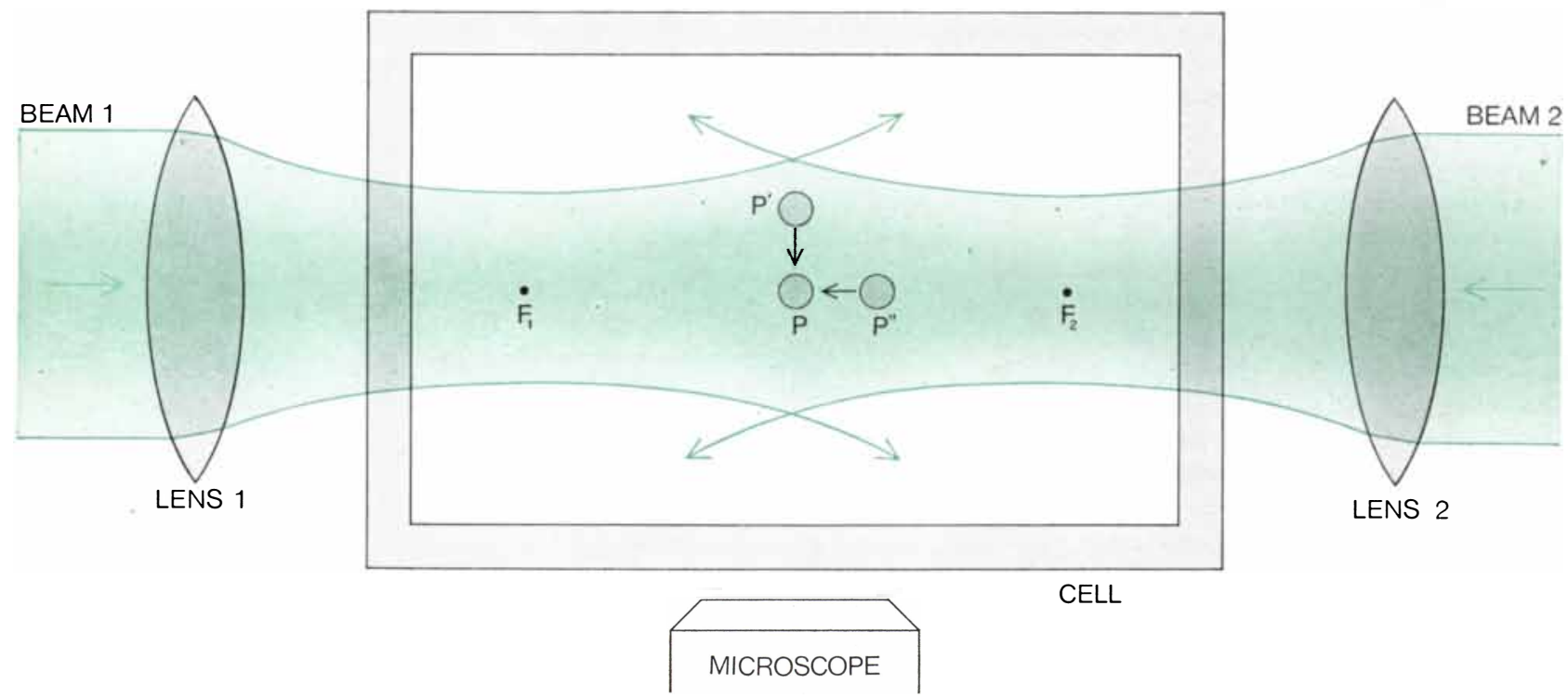

"OPTICAL BOTTLE" shown in this diagram is made up of two opposing Gaussian-mode laser beams. Points $F_{1}$ and $F_{2}$, represent the foci of beams 1 and 2 , that is, the points where the beam diameters are a minimum. Beyond the foci the beams spread by diffraction. A sphere placed at the symmetry point $P$ is in stable equi. librium since any displacement results in an optical restoring force. At $P^{\prime}$ the transverse forces of each of the two beams push the par. ticles back to $P$. At $P^{\prime \prime}$ the force of beam 1 decreases because of the spreading of that beam; beam 2 is more concentrated, however, and hence its force increases, pushing the particle back to $P$. 
ent particles will travel at different velocities in the light beam and thus can be separated. I have already described an instance of the separation of .5micron plastic spheres from 2.5-micron spheres based only on their difference in size. If one wishes to separate biological specimens such as viruses, macromolecules or cells in liquid, the separation would depend not only on their size but also on factors such as the index of refraction and the shape and orientation of the objects. Thus this approach to particle separation could conceivably be more suitable for particular particles than existing separation techniques based on the use of the ultracentrifuge. In any such application care would of course have to be taken to avoid excessive heating.

The preceding observations apply to a range of particle sizes extending from .1 micron to 100 microns. Accelerations as large as 1,000 or $10,000 \mathrm{~g}$ are possible over part of this range. Interestingly enough, there is no problem in observing particles that are small compared with a wavelength of light. Although they cannot be resolved in an optical microscope, one can still detect the presence of even a single particle by observing the hight scattered out to the side of the main beam by the particle.

Tet us now consider particles in more 1 dilute mediums such as air or even vacuum. In these circumstances particles move more freely and gravity plays a more important role than it does in liquids. In particular a demonstration of an optical bottle in air would in essence be a demonstration of optical levitation, where optical forces physically support a particle. In a sense it would be a fulfillment of a search proposed in 1909 by Peter J. W. Debye for a situation in which radiation pressure was strong enough to neutralize gravity. The proposal was part of Debye's Ph.D. thesis on radiation pressure. As an example of such a neutralization of gravity, Debye imagined the çase of very small particles close to the sun. Levitation in this instance would give only neutral equilibrium. As our experiments show, however, levitation with truly stable equilibrium is possible here on the earth with a simple optical bottle consisting only of a single light beam.

Using the laser-levitation approach, a fraction of a watt of laser light was found to be sufficient to support particles about 20 microns in diameter. The transparent glass sphere was placed on a glass plate located inside a glass enclosure, which served to reduce air currents. A verti-

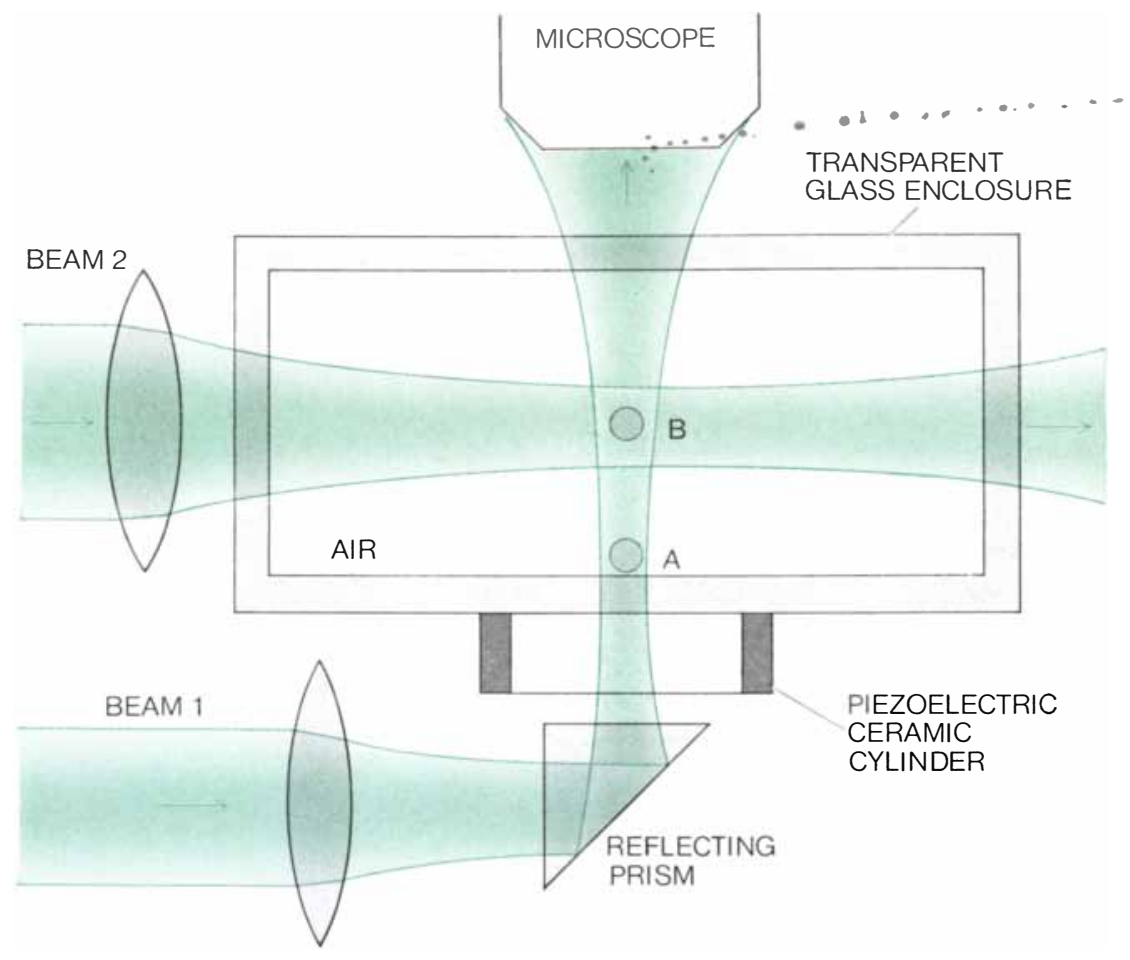

LEVITATION SCHEME employs a single-beam optical bottle. The vertical beam (1) lifts the particle off its resting place at point $A$ and levitates it to point $B$, where radiation pressure balances gravity. In a later experiment a variable probe beam $(2)$ was used to measure the strength of the optical restoring forces by pushing horizontally on the particle.

cally directed beam with a power of about 250 milliwatts was focused on the sphere. The beam exerted a vertical force of several $g$ on the particle. The object was to lift the sphere off the baseplate.

Unfortunately the scheme as described did not work. The trouble was that small particles stick tenaciously to any surface by the forces of molecular attraction known as van der Waals forces. For a 20-micron particle the attractive force is roughly $10,000 \mathrm{~g}$. It was possible, however, to shake the particle loose with a sharp blow to the plate. This suggested a more controlled technique of breaking the van der Waals bond, namely acoustic vibrations. A short burst of acoustic energy coupled into the plate from a piezoelectric ceramic cylinder was enough to momentarily shake the particle loose. Once it was free light pressure took over and the particle began to rise in the diverging vertical beam, coming to rest at the equilibrium point above the plate where radiation pressure and gravity were equal. The particle was levitated!

Since this experiment involved a particle with a high refractive index it was easy to give the particle transverse stability around the equilibrium point. Hence it is possible to achieve levitation with the particle at rest in a position of stable equilibrium. Apart from a technique based on superconducting particles, all other levitation schemes based on electromagnetic forces involve some motion of the levitated particle. In such schemes either the equilibrium is dynamic or some kind of negative feedback is required to correct the particle's position when it moves.

Once a particle is trapped it remains aloft as long as the light is focused on it. By moving the position of the lens one can move the focus of the beam and therefore the position of the particle very precisely. A particle levitated in this way can be photographed by its own scattered light [see illustration on page $62]$.

The levitated particle can also be observed precisely with a microscope focused directly on it. This capability was utilized in an interesting experiment to measure the strength of the optical restoring forces in the optical bottle. By hitting the particle from the side with a second beam it was determined how much power was needed to eventually push the particle out of the bottle [see illustration above]. It was found in this way that the transverse restoring force can be as high as $.5 \mathrm{~g}$ for an off-axis particle when the vertical levitating force is $g$.

The levitation technique thus permits 
the precise micromanipulation of small particles, a capability that is useful in itself. For instance, just being able to introduce a small particle and hold it still as a target for a high-power pulsed laser is important in obtaining laser-generated plasmas for thermonuclear research. By the same token the levitation technique is ideally suited for studying light-scattering from small dielectric spheres, a phenomenon known as Mie scattering. The theory of this type of scattering, developed by Gustav Mie in 1908, bears on the explanation of many phenomena, from rainbows to the angular distribution of light scattered by refractive inhomogeneities in passing through optical transmission mediums. By levitating a single particle of known size and shape

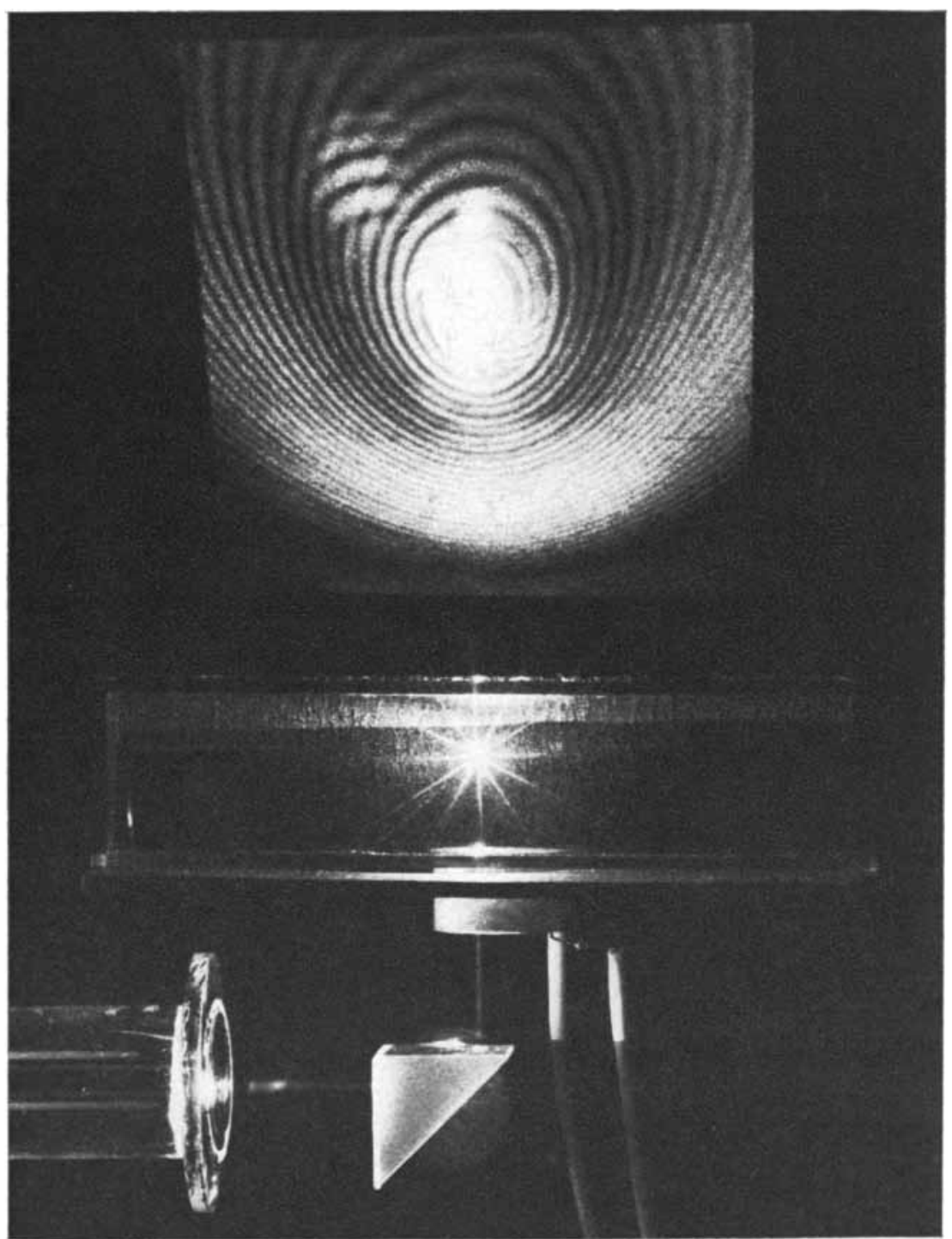

FORWARD MIE SCATTERING of the light from a vertically directed supporting laser beam by means of a levitated dielectric (nonconducting) glass sphere produces a complicated series of interference rings on a screen held at an angle above the levitated particle. The characteristic ring structure associated with this type of light-scattering, originally described by Gustav Mie in 1908, can be seen with unusual clarity in this demonstration. high vacuum. High vacuum eliminates the viscous forces that in air damp out all motions. The optical forces, apart from a minute radiation damping, are frictionless, and a particle set in oscillation or set spinning would continue in this motion indefinitely. Interesting inertial experiments are possible in such instances, perhaps even experiments utilizing the angular, or rotational, momentum of the light. The main problem is how to prevent fluctuations in the laser light from perturbing the particle. So far levitation down to pressures of the order of one torr has been achieved before the particles were lost. At a pressure of one torr the viscous damping is already reduced and oscillations due to perturbations build up and lead to the eventual escape of the particle. Stabilization of the laser power and stronger optical bottles involving several beams are possible and would allow levitation at still lower pressure. A simple optical feedback system based on particle position or velocity, however, might have to be introduced eventually. This would be a way of simultaneously stabilizing laser power and particle position that could be made very sensitive.

Another potential application of radiation pressure involving high vacuum is the acceleration of small neutral particles to high velocity. If a high-power laser beam is applied to a particle, the particle will be accelerated rapidly along the axis of the beam while being trapped transversely by the beam. What, one wonders, limits the ultimate velocity attainable? The answer is probably only the eventual melting and vaporization of the particle. Setting melting as the limit, if one knows the optical absorption coefficient of the particle, one knows how much light one can apply to the particle and one can calculate the final velocity. The best optical glass available has an absorption coefficient of roughly $3 \times 10^{-5}$ per centimeter. (This number is important for optical communication through glass fibers and much work is currently being done to decrease it.) Using this value, one gets a final velocity of about $3 \times 10^{8}$ centimeters per second for a .5-micron particle. That is an incredible velocity for a macroscopic particle. If such a particle were to strike a target or another particle of the same size and velocity, one would obtain a pulse with a power of some $10^{11}$ watts for about $10^{-13}$ second. The particle would vaporize and form a plasma whose temperature would be some 50 times higher than the temperature needed for thermonuclear reactions in deuterium. This approach might therefore be 
of interest in thermonuclear research and other areas where short high-power pulses are important. The apparatus for such a particle accelerator is well within present technology. Unfortunately it might end up almost as long as the twomile Stanford linear particle accelerator because of the need to keep the intensity low enough to avoid nonlinear absorption. Such an experiment would be no small undertaking.

Thus far I have described the effects of concentrating laser light on small nonabsorbing particles with a diameter in the range between .1 and more than 100 times the wavelength of the light. If we consider really small particles such as atoms, which are a few thousandths of a wavelength in diameter, we might expect negligible pressure effects, since waves tend to diffract around such small objects without much deviation. Although light does generally pass through gases with little interaction, there are important exceptions. To appreciate these special cases one must resort to quantum theory, which is the basis of our present understanding of light and matter. Light, in addition to being a wave phenomenon, is known to have a particulate nature. A light beam is revealed as the sum of the irreducible units of energy known as photons, each with a definite energy and momentum. Likewise the atom is shown to be a resonant structure that can exist only in particular energy states. Atoms interact strongly with light when the energy of the photon is just enough to change the atom from one distinct energy state to another. This is a resonance phenomenon. A measure of the strength of the resonant interaction is a quantity called the absorption cross section. The idea of an absorption cross section is based on the concept that any photon striking this area is absorbed. For atoms "on resonance" the absorption cross section is roughly equal to the wavelength squared. Since one can focus laser light to a spot having a theoretical area almost equal to the wavelength squared, this finding implies that a single atom properly placed at the focus of such a beam will absorb almost all the light in the beam. This turns out to be essentially true for low intensities where saturation effects are absent. In any case resonant atoms are the most absorbent material known for light. This fact implies large radiation-pressure effects.

In the absorption process both the energy and the momentum of the photon are delivered to the atom. Consider, for example, a sodium atom originally in its ground, or lowest-energy, state, which
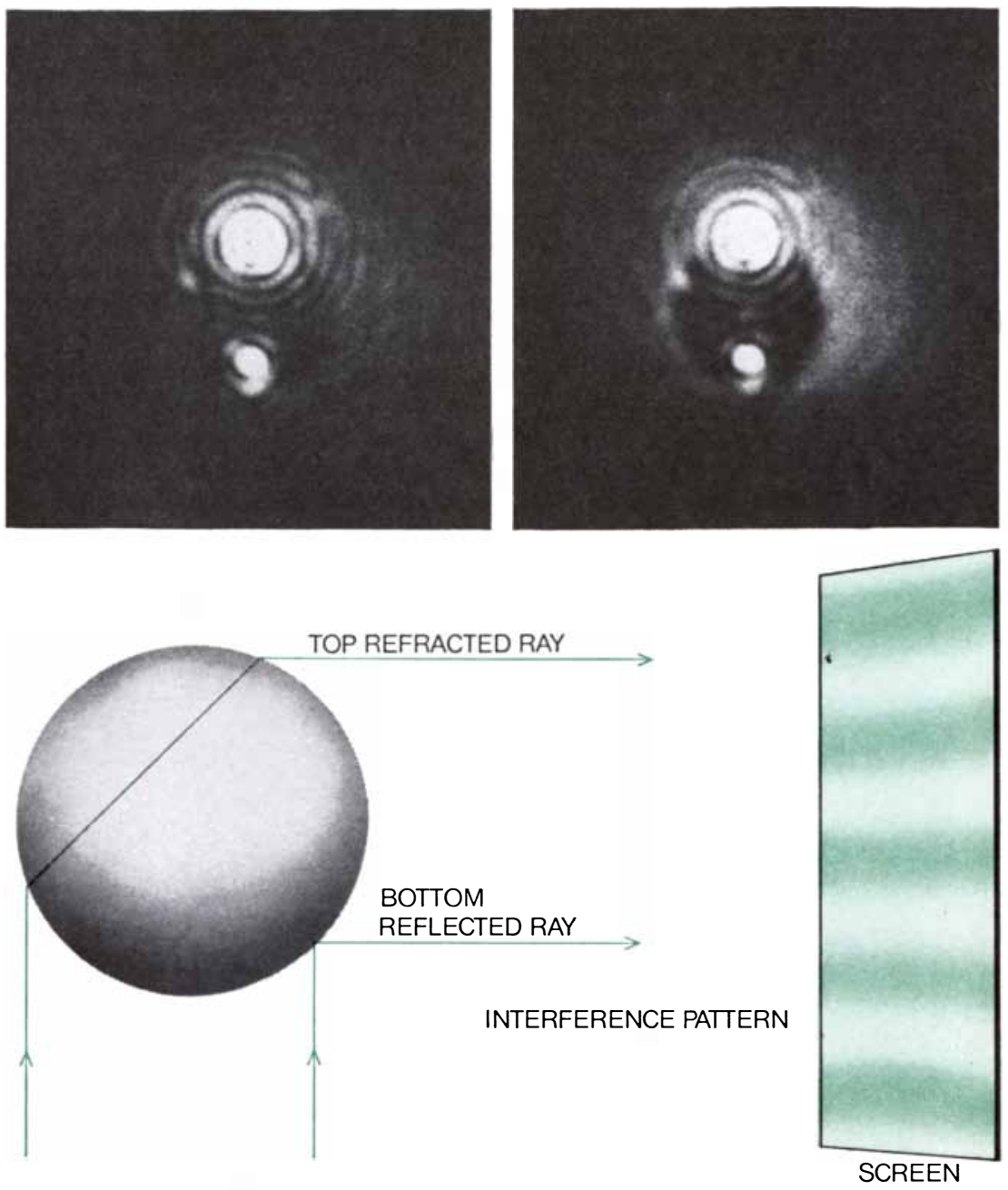

TWO SEPARATE BRIGHT SOURCES appear when a levitated particle is viewed at an angle of 90 degrees by its own scattered light with a microscope focused on the particle (top left). With a light background added behind the particle (top right) one can more readily see the outline of the particle and the location of the sources with respect to it. The diagram at bottom explains the origin of the two sources at 90 degrees. One refracted ray emerges close to the top of the particle, while a weaker reflected ray bounces off the particle somewhat above its bottom edge. These two sources interfere to give the horizontally striped far-field interference pattern observed on a screen located a long distance away.

absorbs a photon of yellow " $D$ line" resonance radiation. The atom not only is raised to an excited state but also receives an increment in velocity of about two centimeters per second in the direction of the incident light. The atom remains in the excited state for an average time, called the excited-state lifetime, before it returns to the ground, or absorbing, state. It does so spontaneously with the emission of a photon of the same frequency in a random direction. This gives another randomly directed impulse of two centimeters per second to the atom. Subsequent absorptions and spontaneous emissions give additional directed impulses along the beam followed by random impulses. The result is a net force directed along the light beam. The effect is similar to the force a water hose exerts on a ball that scatters the stream in all directions.

This optical force, called resonance radiation pressure, has some unique features. First, it does not keep on increasing with increasing optical power but rather becomes saturated at a fixed maximum value that is related to the finite lifetime of the excited state for spontaneous emission. If the average lifetime is about $10^{-8}$ second, as it is for sodium, one might expect the atom to be capable of scattering at most $10^{8}$ photons per second. Actually it can scatter only half that amount, for reasons associated with stimulated emission from the excited state back down to the ground state. In this process, which occurs at high light intensities, the atom is stimulated to give a photon back to the beam. That by it- 


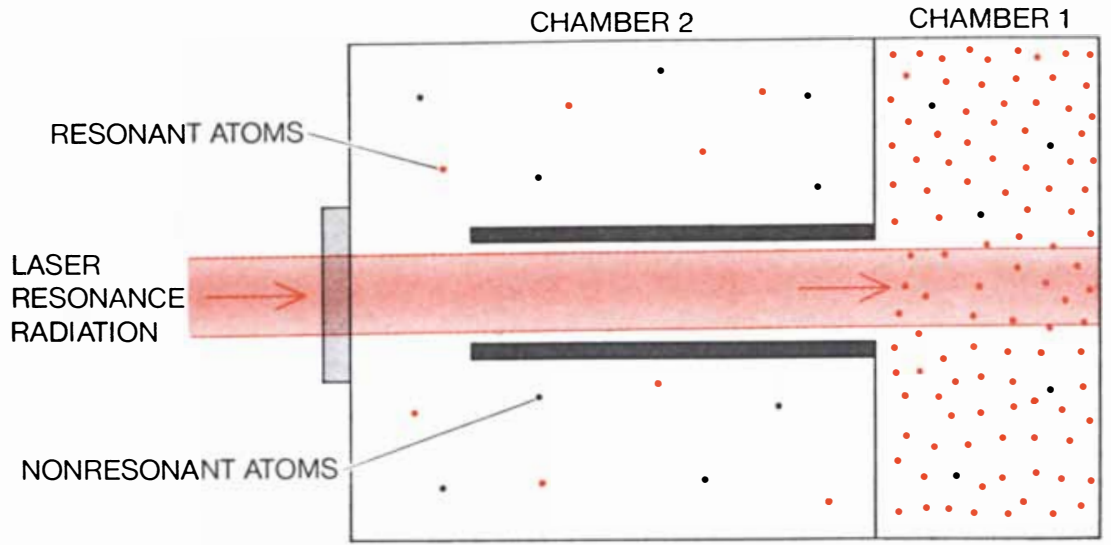

GASES CAN BE SEPARATED by the resonance radiation pressure of laser light. In this proposed scheme the laser light acts as a pump, pushing most of the resonant gas atoms (colored dots) into chamber 1 without affecting the nonresonant gas atoms (black dots).

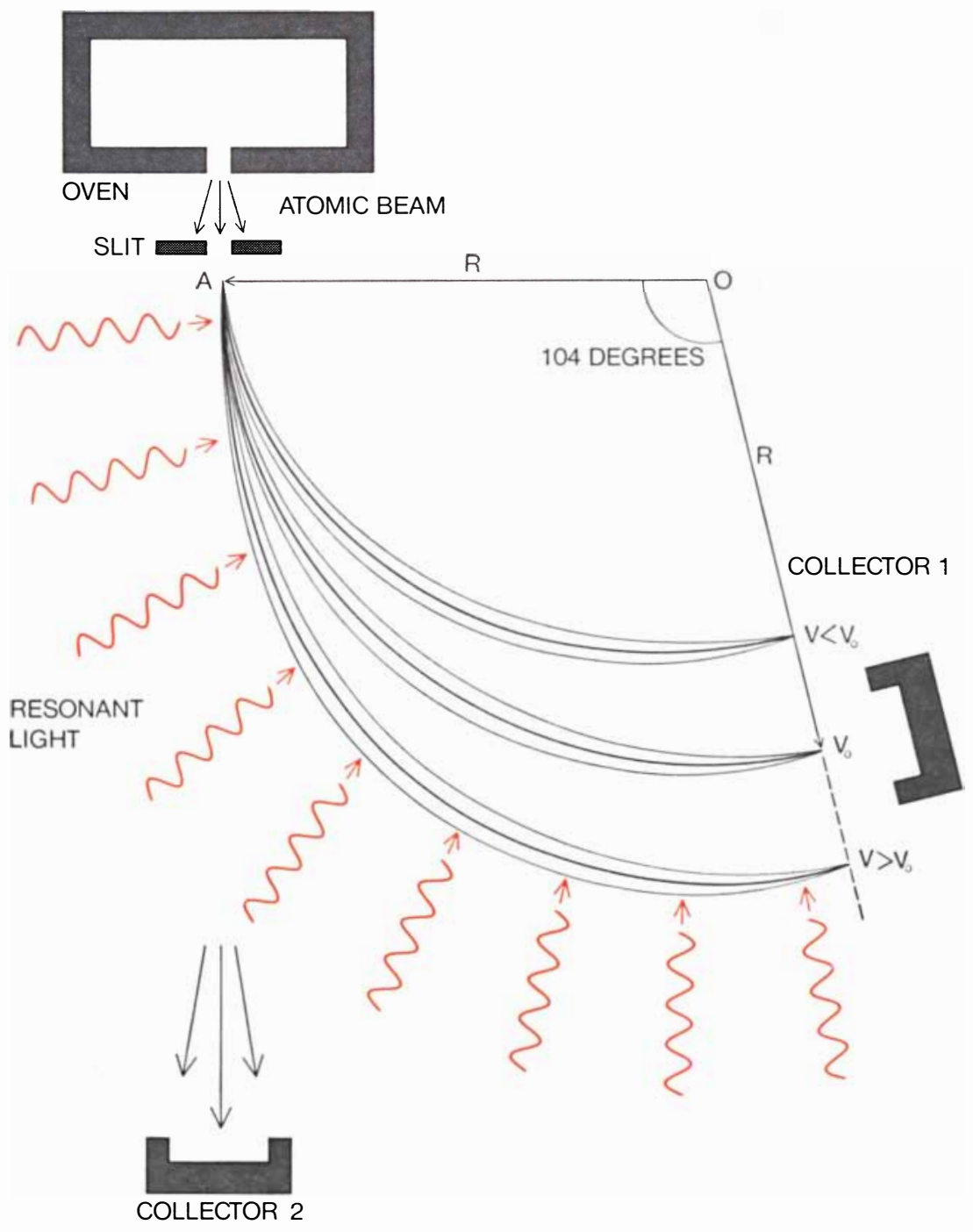

ATOMIC-BEAM VELOCITY ANALYZER employing resonant laser light would make it possible to select atoms of a particular velocity from a multivelocity beam. The resonance light directed radially inward toward point $O$ is perpendicular to a circular orbit of radius $R$. Collector 1 detects refocused atoms of different velocity at different radii after they are deflected through an angle of 104 degrees. Unaffected nonresonant atoms go to collector 2. self contributes negligibly to the force but results in an atom's spending only half as much time in the excited state, from which it can decay randomly by spontaneous emission. The net result, however, is a saturated resonance-radiation-pressure force of some $100,000 \mathrm{~g}$ on the atom.

The other unique feature of this effect is the need for exact resonance. Consider a laser beam tuned to resonance for an atom at rest. If the atom begins to move in the direction of the light because of resonance radiation pressure, then as a result of the Doppler shift it will no longer be in strict resonance and the force will decrease.

One possible use of this force is the separation of gases, with light as an actual pump that operates selectively on resonant atoms [see top illustration at left]. Suppose one fills a double-chambered vessel with two gases and shines light that is resonant with only one of the gases down the open pipe connecting the chambers. The constant saturated force on the resonant atoms causes an exponential pressure distribution along the pipe starting at some high value in the far chamber and falling to a low value in the near chamber when the system finally comes to equilibrium with the light force. The nonresonant gas remains unaffected by the light. The gases could thus be separated in this way, which is analogous to the way the constant force of gravity separates gases of varying weights into different exponential pressure distributions in the earth's atmosphere. With resonance radiation pressure even different isotopes of the same atom could presumably be separated by virtue of the isotope shift in the frequency of the resonance. Separation of isotopes is often difficult because of the similarity in chemical properties of isotopes of the same atom.

ther possible applications of radiation-pressure techniques involve atomic and molecular beams. Much fundamental knowledge has been obtained from such beams by studying their deflection in static fields and their interaction with electromagnetic waves and other particles. It should now be possible using the large forces of radiation pressure to optically deflect atomic beams through large angles and thus study them by a new means. One possible velocity-analyzer scheme selects atoms of a particular velocity from a multivelocity beam, with resonance radiation focused to a point to establish a uniform centralized optical force field [see bot- 
tom illustration on opposite page]. Such a constant radial force is possible even though the light intensity varies with position owing to the saturation property of the force. This field has the interesting property that all resonant atoms injected at a certain point with a particular velocity refocus themselves to a point after being deflected by 104 degrees, but at a radius that depends on the velocity. Such large deflections free of Doppler shifts are possible because all the atoms move in orbits close to a circle passing through the input point. Along the circle the constant force of radiation pressure and centrifugal force exactly balance and no motion occurs in the direction of the light. The situation is analogous to the familiar motion of a particle rotating at the end of a string with constant tension. Thus if a collector is placed at an angle of 104 degrees, it can collect atoms of different velocity at different radii. Any nonresonant species present in the original beam are undeflected and pass to another collector. To make a velocity selector of this type in which a beam of sodium atoms emerging from a heated oven is bent around a radius of five centimeters would require about a watt of resonant light. If one were to extend the converging light beam completely around the circle and add some convergence to the light along the axis of the field, one can conceive of trapping resonant atoms stably in a closed circular orbit [see illustration at right]. Such an arrangement would then be, for atoms, the closest analogue to the optical bottle for macroscopic particles. The implementation of these applications of radiation pressure to atoms calls for laser sources of sufficient power and tunability. The rapid strides being made in tunable dye lasers and tunable parametric oscillators will, one hopes, make such experiments possible.

Many aspects of this work have clear historical precedents. The recoil of the electron in a single high-energy photonelectron collision, known as the Compton effect, is an example of radiation pressure (although it is not a resonant interaction). In more closely related work the minute recoil of neutral sodium atoms after absorbing and emitting a single photon from a weak resonance lamp was observed by Otto R. Frisch in an extremely sensitive atomic-beam experiment in 1939. The idea evidently extends back to Einstein, since Frisch called his achievement the observation of the "Einstein recoil." More recently evidence of resonance radiation pressure on a cosmic scale has been discovered

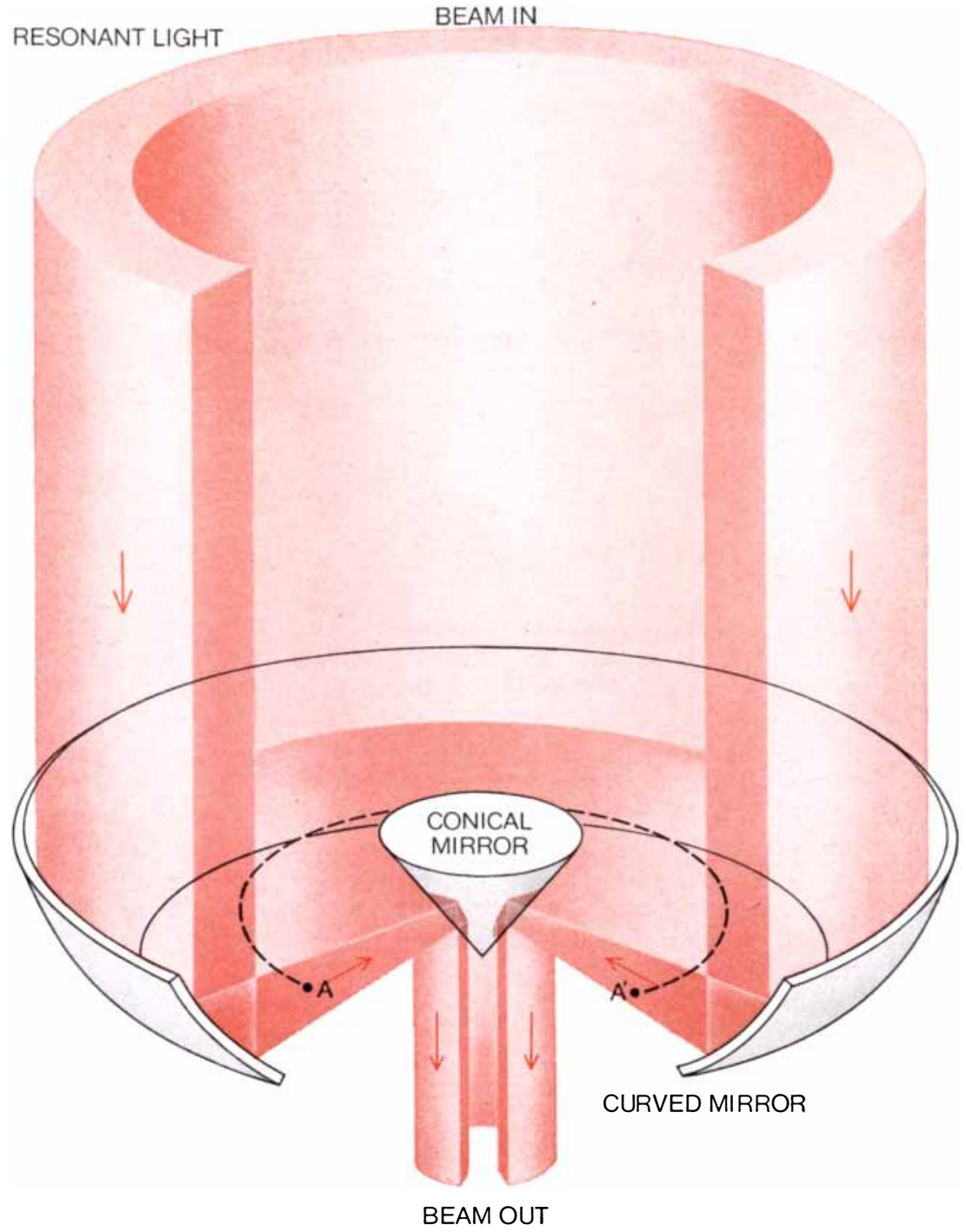

HYPOTHETICAL APPARATUS for optically trapping resonant atoms in a stable circular orbit $\left(A A^{\prime}\right)$ would be analogous to the optical bottle for macroscopic particles. The concept is a variation of the velocity-analyzer scheme, with the converging light beam ex. tended around the circle and with some convergence added to the light along axis of field.

in astronomy. From observations made on far-ultraviolet resonance lines with rockets shot above the atmosphere, Donald C. Morton of Princeton University has concluded that large quantities of gas are being ejected from very hot stars at velocities as high as $2 \times 10^{8}$ centimeters per second. Leon B. Lucy and Philip M. Solomon of Columbia University attribute this phenomenon to the fact that the resonance radiation pressure of the star's intense ultraviolet thermal radiation can greatly exceed gravity for various ionized atoms. Owing to the continuous nature of the thermal radiation, Doppler effects do not reduce the driving force as the velocity of the ejected atoms increases. The forces involved are still much less than the saturated forces one can hope to obtain with lasers.

In another area G. S. Kutter and Malcolm P. Savedoff of the University of Rochester suggest an explanation of the origin of planetary nebulas in terms of radiation pressure. In their view planetary nebulas (the gas clouds observed around many white-dwarf stars) are thought to result from flare-ups of the star's surface temperature.

Clearly the history of the study of radiation pressure has been closely related to the history of science itself. This association, however, is merely an example of how the entire structure of science is inextricably bound together. New knowledge and techniques discovered in one area are inevitably related to new knowledge and techniques in other areas. 